\title{
Research on the Current Situation and Countermeasures of the College Laboratory Safety Management
}

\author{
Lan Chen ${ }^{1,}$, Jinglun Yang ${ }^{2, b}$ \\ ${ }^{1}$ Laboratory and assets management office,Zhuhai College of Jilin University, Zhuhai 519041,China \\ ${ }^{2}$ Sports education center,Zhuhai College of Jilin University,Zhuhai 519041,China \\ achenlan19841009@163.com, ${ }^{b}$ yangjinglun1983@163.com
}

Keywords: college laboratory, safety management, countermeasures .

\begin{abstract}
The college laboratory plays an important role in the teaching activity and scientific research, which it is considered as an important place of talent cultivation and technological innovation. The improvement of the college laboratory safety management is a persistent goal for the college laboratory administrators. In this paper, the realistic background for the rapid development of the higher institutions in recent years is illustrated. The major problems include the lack of the sufficient laboratory technical personnel and safety management ability; insufficient capital investment on the laboratory safety management; unsound laboratory safety management system and lack of practical significance; outdated information management. The corresponding countermeasures are proposed; the construction of the scientific and effective laboratory safety management mode is designed to unceasingly improve the college laboratory safety management level.
\end{abstract}

\section{Introduction}

The college laboratory is an important place of technological innovation and talent cultivation. In recent years, the higher institutions are developed in a rapid way. Thus the scale of the college laboratory is unceasingly extended; the laboratory has more and more users; the laboratory equipments are increased; the using area per capita is decreased. As a consequence, the security issues of the college laboratory are gradually highlighted ${ }^{[1]}$.

The colleges shall actively explore the suitable and practical management mode so as to efficiently handle with the existing laboratory safety issues and improve the safety management level. However, there are many adverse factors like the lack of the sufficient laboratory technical personnel and safety management ability; insufficient capital investment on the laboratory safety management; unsound laboratory safety management system and lack of practical significance; outdated information construction. Consequently, the contradictions between the growing demand of the laboratory software and hardware construction and outdated safety management are deepened. In recent years, the safety accidents are frequently raised. Since the laboratory safety management becomes more and more serious, the college shall attach great importance to the construction of the laboratory technical personnel, improve the safety management ability, increase the capital investment on the laboratory safety management, fulfill the construction and management of the laboratory safety system, take advantage of the modern information technology, enhance the safety management, analyze the existing issues of the laboratory safety management, actively explore the suitable management countermeasures under new situation and provide a secure learning environment for the teachers and students.

\section{Analyze the major issues of the college laboratory safety management}

\subsection{Lack of the sufficient laboratory technical personnel and safety management ability.}

The laboratory technical personnel are the major undertaker of conducting the teaching activity, experimental equipment maintenance and laboratory safety management ${ }^{[2]}$. Under the current 
situation, the usage frequency of doing experiments is increased; the scale of the laboratory is higher; the functions of the laboratory are diversified. At the same time, the laboratory technical personnel are not correspondingly increased. With the lack of the laboratory technical personnel, many problems are raised. For instance, one administrator has to simultaneously manage a few of the laboratories, keep the good state of more expensive laboratory equipments, have no time to join in the professional training and take the advanced study. The laboratory management tasks are arduous; there is less time of making the training and self-improvement. It is unable for the administrators to deepen the learning on the usage and maintenance of the laboratory equipments, make a comprehensive understanding on the laboratory management tasks and improve the safety management ability. During the daily management process, the laboratory administrator even can't immediately find out the problems and solve the problems, which it has greatly restricted the working progress of the laboratory safety management.

\subsection{Insufficient capital investment on the laboratory safety management.}

Since there are not sufficient capital investments, the colleges generally attach great importance to the input benefits. Therefore, a large proportion of the funds are usually allocated to the teaching area and scientific research field. There are not enough capital investments on the daily management of the college laboratory safety management ${ }^{[3]}$. The major disadvantages are illustrated as follows: (1)the laboratory construction can not keep pace with the expansion speed of the college enrollment; thus there are many existing problems such as shortage of the laboratory room, small laboratory space, short distance of experimental operation and blockage of the safety passageway; (2)there are no enough fire-fighting equipments; some fire-fighting equipments are worn down by the years. As these equipments are not properly replaced, it has brought about many potential safety hazard.(3) While the laboratory is purchasing new equipments all the time, the old equipments are not completely scrapped; thus the narrower laboratory room will give rise to the huge safety hazards in the laboratory.

As the scale of the college laboratory is constantly expanded, it is necessary to increase the laboratory safety management funds. When there are not sufficient capital investments, it is unable to fulfill some laboratory safety management tasks. In this way, it is liable to bring about the security accidents. According to the investigation results, more than $90 \%$ of security accidents are correlated with the lack of the laboratory administrator's prevention awareness. The prevention awareness of the laboratory administrators has a close correlation with the college administrator's attitudes. Obviously, the college leadership has to pay enough attention to the laboratory safety management ${ }^{[4]}$.

\subsection{Unsound safety management system and lack of practical significance.}

The laboratory safety management is involved with all kinds of professional areas like fireproofing, theft-proof, biological toxicity, electro-mechanical accidents, disposal of dangerous chemical waste, etc. Only the sound and standardized management system can solve these problems. At present, the colleges have formulated the corresponding regulations on the laboratory safety management methods, implementation rules, safety responsibility system and contingency plan . However, these rules and regulations are made a few years ago, which these rules and regulations are lack of the prospective and practical significance. These disadvantages are stated as follows: the theoretical rules and regulations are lack of detailed descriptions and practical significance; thus it is much difficult to give the effective guidance on the implementation of the laboratory safety management system; (2)the old system can not keep pace with the times, meet the new requirements for the safety construction of the college laboratory; (3) in terms of the system, the people-oriented management concept is not clearly expressed. In particular, the system is generally formulated based on the role of the teachers. Consequently, it is unable to reflect the student's dominant role on the laboratory safety management.

\subsection{Outdated information construction.}

Since the college laboratory safety management starts relatively late in China, there are no strong management theory basis. According to the research results, the information construction of the college laboratory safety management is not favorable. With the further progress of the college 
laboratory construction and management, the disadvantages of the former safety management modes are highlighted, which it is impossible to meet the new requirements of the contemporary laboratory safety management. For instance, the conventional management mode and recording methods have reduced the college laboratory administrator's working efficiency; the insufficient and delayed information communications have increased the laboratory safety personnel's working strength; at the same time, the service level of the laboratory is also reduced; the outdated information has imposed restrictions on the college safety culture and improvement of the student's safety consciousness. These factors are not beneficial to standardize the daily teaching activity and scientific research, improve the usage rate of the laboratory room and enhance the laboratory safety management level. The existing problems of the college laboratory safety management are still to be solved.

\section{Propose the solutions on the laboratory safety management}

\subsection{Focus on the organization of the laboratory technical personnel and improve their safety} management ability.

The laboratory technical personnel is an indispensable or important component on the college teaching activity, scientific research, laboratory construction and management. Influenced by the conventional concepts, the domestic college leadership generally consider the laboratory technical personnel as the auxiliary teaching staff. The organization of the laboratory technical personnel is not highlighted $^{[5]}$. In the new era, the college leadership shall renew their ideas, approve the important role of the laboratory technical personnel, focus on the organization of the laboratory technical personnel. In particular, it is necessary to strength the training of the technical personnel and improve their management ability. On one side, the college leadership shall systematically reduce the workload of some laboratory technical personnel in batches. The laboratory administrators are encouraged to take advantage of the leisure time, take the self learning and improvement in many ways, constantly complement and update the knowledge, expand the technical field and knowledge structure and enhance the laboratory safety management ability; on the other side, the college leadership shall create the opportunity for the laboratory technical personnel to receive the further education, training and communication and popularize the laboratory safety management knowledge. In this way, the laboratory administrators will have a basic understanding and new recognition on the laboratory safety, transform the outdated management concept, establish the correct safety awareness and guarantee the normal operation of the laboratory.

\subsection{Increase the capital investment on the laboratory safety management.}

The laboratory hardware facility is fundamental to guarantee the safety of the college laboratory. Thus it is urgent for the colleges to focus on the safety construction of the college laboratory, increase the capital investment, promote the standardized installation of the laboratory hardware facility. The colleges shall do well with the laboratory safety management from the following aspects: (1)the colleges shall keep pace with the expansion speed of the college enrollment, increase the capital funds, expand the construction of the college laboratory, increase the laboratory area and solve the problems of the crowded space; (2) it is essential to strengthen the construction of the laboratory safety infrastructure. These infrastructure shall conform to the national security criteria concerning the emergency exits, fire-fighting equipment, fire-alarming system, surveillance system; (3) in order to guarantee the security of the users, it is necessary to properly update and dispose the obsolete devices so as to create the secure and comfortable teaching environment.

\subsection{Fulfill the establishment and management of the laboratory safety system.}

The sound laboratory safety management system is essential to guarantee the normal laboratory operation and do well with the daily safety management ${ }^{[6]}$. With regard to the laboratory safety management, the laboratory apparatus and human resources shall be well developed with the sound laboratory management system. During the process, the college shall adhere to the "teacher-led and student-centered " disciplines, deepen the people-oriented management concept, formulate the comprehensive laboratory safety system, properly update and fulfill the laboratory management 
system, adapt to the contemporary requirements of the laboratory safety management and improve the standardized and practical significance of the laboratory management system. In addition, the college is responsible to monitor the implementation progress based on the established laboratory safety management rules and regulations. The effective implementation of the safety management system can improve the safety management efficiency and service quality of the college laboratory.

\subsection{Enhance the laboratory safety management based on the information technology.}

In the new era, the college leadership shall find new methods of fulfilling the laboratory safety management, actively explore the advanced information technology, maintain the stable operation of the laboratory, create the favorable environment on the experimental teaching activity and scientific research. To begin with, it is necessary to take advantage of the information technology, set up the laboratory safety assessment system and carry out the laboratory safety rules and regulations. Before entering the laboratory, the teachers, students or administrators or other related personnel shall participate in the training and examination of the laboratory safety knowledge. Only the laboratory personnel pass the examinations can she/he join in the learning and working activities in the laboratory. Secondly, based on the advanced network and information technology, the QR code is available for anyone to access the laboratory information page at any place and any time,properly understand the related news release and improve the laboratory administrator's working efficiency. Thirdly, it is able to establish the college website, publicize the laboratory safety culture, organize and develop the safety culture, enhance the teachers and student's safety consciousness and improve their comprehensive security awareness.

\section{Summary}

The college laboratory safety management is an important component of ensuring the normal operation and development of the teaching activity and scientific research. The safety management level of the college laboratory has become the important indicator of judging the level of the college teaching activity, scientific research and laboratory management ${ }^{[7]}$. As the laboratory safety accidents are occasionally raised in recent years, the college has to shift the laboratory construction works into the laboratory safety management. However, the laboratory safety management is a long-term and complex project. During the process, the college leadership shall pay attention to the organization of the laboratory technical personnel, set up the comprehensive safety management system, increase the safety management capital investment and explore the advanced information technology. The standardized laboratory safety management mode is of great importance to create the secure and healthy teaching environment, guarantee the normal operation of teaching activities and scientific research, deepen the talent cultivation, scientific research and social service function.

\section{References}

[1]Li Ying, Make the reform on the laboratory safety management system and adapt to the innovative experimental teaching needs, Research and Exploration in Laboratory ,2015,11:280-283.

[2]Zhang Wenjun, Research progress and practices of the college laboratory safety management, Research and Exploration in Laboratory,2016,01:282-285.

[3]Qi Wentao, Sun Shuqiang, Fan Bing, Develop the college laboratory safety culture based on the information technology, Research and Exploration in Laboratory,2016,02:295-299.

[4]Gu Hao, Cao Qun, Sun Zhijie, Li Xiuzhen, Fulfill the laboratory safety education system, Research and Exploration in Laboratory,2016,04:281-283+292.

[5]Huang Kun, Li Yanqi, Analyze the current situation and countermeasures of the college laboratory safety management in China, Research and Exploration in Laboratory,2015,01:280-283.

[6]Song Zhijun, Wang Tianshu, Cai Meiqiang, Xie Hujun, Analyze the current situation and 
countermeasures of the college laboratory safety education, Research and Exploration in Laboratory,2015,08:280-283+288.

[7]Cui Guanxun, Discuss on the laboratory safety management system design based on the Internet of things technology, Research and Exploration in Laboratory,2015,03:287-290. 